\title{
Hybrid WiFi-WiMAX Network Routing Protocol
}

\author{
${ }^{1}$ Susana Rivera Ibáñez, ${ }^{1}$ Raúl Aquino Santos, ${ }^{2}$ Victor Rangel Licea, ${ }^{1}$ Arthur Edwards Block, \\ ${ }^{1}$ Miguel Ángel García Ruiz \\ ${ }^{1}$ College of Telematics, University of Colima, C.P. 28040, Colima, México \\ ${ }^{2}$ Department of Telecommunications, UNAM, C.P. 04510, México, D.F. \\ aquinor@ucol.mx, susana rivera@ucol.mx, victor@,fi-b.unam.mx, \{arted, mgarcia\}@ucol.mx
}

\begin{abstract}
The proliferation of Wireless Local Area Networks (WLANs) facilitates the development of multihop routing protocols. Moreover, the need to cover greater areas has led to the creation of new standards for Wireless Metropolitan Area Networks (WMANs). This paper proposes a new routing protocol that integrates WLANs and WMANs, allowing seamless interconnectivity.
\end{abstract}

\section{Introduction}

Wireless technology has greatly evolved to meet the exponential growth of mobile devices in the last two years [1]. Although access to wireless technology has grown, there are still significant limitations that make it difficult for some to exploit its proven and potential benefits. The main difficulty regarding expanded coverage is that WiMAX (Worldwide Interoperability for Microwave Access) broadband wireless technology is not available in all regions. WiMAX, precisely speaking, represents the future of the broadband wireless communications because it offers a variety of services to users, regardless of location.

One important strategy to gain greater access to wireless services is to employ multihop Wireless Fidelity (WiFi) networks to access WiMAX technology through a gateway. Although the attempts of interconnectivity of WiMAX and WiFi technologies have existed for several years, there are still many challenges to seamlessly exploit its potential.

In [2], Rabindranath et al. present a performance analysis of a university network combining the IEEE 802.11n standard and WiMAX technology. The paper describes the benefits of using these technologies in tandem, even when one of them is recently approved (WiMAX) for certifying purposes and the other is scarcely commercially available $(802.11 \mathrm{n})$. The document proposes possible solutions for problems related to the integration of these two technologies. However, the authors themselves recognize that security issues and the lack of availability of adequate equipment for testing the network's performance were significant limitations.

In [3], Shilpa et al. present a comparative study of emerging WiMAX, 3G, and WiFi wireless technologies. The authors describe the main characteristics of these technologies, as well as the advantages and disadvantage of each of them. Their paper, however, is theoretical in nature and does not provide quantitative results based on simulations.

Currently, WiFi-WiMAX integration is based on the IEEE802.16d protocol, also known as Fixed WiMAX. The IEEE 802.16e standard has yet to be deployed because it is still undergoing the certification process. One of the greatest difficulties to be solved is how to deal with quickly changing network nodes that can cause breaks in the network connection. Consequently, any proposed routing algorithm must allow for highly dynamic nodes and network partitions.

The rest of the paper is organized as follows. In Section 2, the theoretical bases of WiFi and WiMAX technologies are introduced. Section 3, describes the proposal of the routing protocol and terms of flow diagrams. Finally, Section 3 resents results and future research.

\section{Theoretical Foundation}

The three types of wireless networks can be classified according to their coverage areas:

(a) WPAN (Wireless Personal Area Network), where every device in the network can communicate with other using a personal area network. Bluetooth is an example of this technology. 
(b) WLAN (Wireless Local Area Network), where the devices communicate among themselves by means a centralized Access Point (AP) or base, and

(c) WMAN (Wireless Metropolitan Area Network) which provides higher transmission speeds and covers greater areas, efficiently exploiting bandwidth while minimizing interference [4].

Currently, the access to the Internet using wireless technology at homes, offices or college campuses has become common and has displaced traditional cable technology because its relatively low cost, mobility and high data transmission speeds [5].

The integration of WLAN (Wireless Local Area Network) and WMAN (Wireless Metropolitan Area Network) will benefit both users and service providers. Users will benefit from the performance of expanded network coverage and greater data transmission speeds. Economically, service providers will receive a better return on their investment because of the larger number of users because this technology covers a greater area and provides better services [6].

\section{Theoretical basis of WiFi and WiMAX}

\subsection{WiFi}

WiFi is a trade mark of the WiFi Alliance (Wireless Ethernet Compatibility Alliance), which is in charge of testing and certifying the interoperability of devices based on the IEEE 802.11 standard [3]. WiFi allows APs a coverage radius, or hotspot, of approximately $100 \mathrm{~m}$ in interior spaces [3]. Meanwhile, WiFi exterior coverage can cover a radius of approximately $300 \mathrm{~m}$, depending on environmental conditions [7]. The transmission speed of the different WiFi standards ranges from $11 \mathrm{Mbps}$ until 54Mbps [3].

WiFi's advantages include:

- its use of a non-licensed frequency band.

- its fewer international regulatory restrictions.

- its infrastructureless architecture that allows for ubiquitous functioning and dynamic growth.

- its low cost.

- its mobility without network connection breaks.

WiFi's disadvantages include:

- its use of the $2.4 \mathrm{GHz}$ spectrum, which is susceptible to interference.

- Its higher energy consumption when compared to other standards.

\subsection{WiMAX}

WiMAX is the acronym for Worldwide Interoperability for Microwave Access and its architecture is based on broadband point-to-multipoint wireless access [3].

WiMAX was created in 2001 to promote the IEEE 802.16 standard. This standard was finally approved in June 2004 [4]. The 802.16 standard provides for fixed and mobile WiMAX in 802.16d and 802.16e, respectively [9]. Some important characteristics of WiMAX include:

- its use of the microwave frequency band for wireless data transmission

- its high transmission speed over long distances.

- its use of OFDM (Orthogonal Frequency Division Multiplexing) to enable non-line-of sight communication.

- its multi-channel support for TDD (Time Division Duplex) and FDD (Frequency Division Duplex)

- its flexible handling of channels in the $3.5 \mathrm{MHz}, 5 \mathrm{MHz}$ and $10 \mathrm{MHz}$ frequencies.

Some challenges for WiMAX include:

- reaching a coverage area of up to 10 miles.

- providing wireless broadband and dedicated links.

- making the technology more affordable.

- allowing access from more remote areas.

Although WiMAX technology is relatively new, its brief history and development consists of four phases [8]:

In December, 2001, the Wireless MAN-SC standard was established. This standard specifies the physical layer and multichannel techniques, including the single-carrier that can handle both TDD and FDD. In 2003, WiMAX was consolidated under the IEEE 802.16a standard to support OFDM in the PHY layer. During this time substantial changes were made to the 802.16a standard, resulting in the 802.16c standard. The 802.16c standard is the basis of HIPERMAN (High Performance Metropolitan Area Network); and of 802.16e-2005, which specifies scalable OFDM for the PHY layer.

As already mentioned, the WiMAX standard is divided into several sub-standards:

802.16a: The original standard which allows for a $70 \mathrm{Mbps}$ speed at distances of up to 30 miles using the $10 \mathrm{GHz}$ and $66 \mathrm{GHz}$ bands. 
802.16e: This standard is the newest standard and employs the $2 \mathrm{GHz}$ and $6 \mathrm{GHz}$ bands. This standard allows mobile devices to use wireless technology.

The 802.16e-2005 standard was developed under IEEE guidelines, but the implementation was left to private industry. The WiMAX Forum was created to solve interoperability problems and promote the standard itself [8].

The WiMAX architecture is very similar to a cell phone network in that the service area is divided into cells that enable it to function with or without line of sight (LOS), as it is based on point-to-multipoint broadband wireless access.

One important characteristic of WiMAX is that it uses OFDM, which is based on modulation by multiple carriers and Frequency Division Multiplexing (FDM). In OFDM, the bandwidth is divided into parallel subcarriers with different channels. OFDM uses subcarriers that are mathematically orthogonal and the information is sent in parallel to the different subchannels.

WiMAX is not the only solution for broadband wireless services. Private consortiums such as i-Burst by ArrayComm and Flash-OFDM by Flarion, both of which are partially compatible with WiMAX [8], also exist in the marketplace. Other systems include the $3 \mathrm{G}$ for cell phone and WiFi for Local Area Network (LAN) [8].

For WiMAX to be successful, it needs to provide better performance than $3 \mathrm{G}$ and WiFi. Some of the challenges facing WiMAX include:

- improving the signal propagation mechanism because using the atmosphere as the transmission medium has several problems.

- $\quad$ increasing the range of band frequencies to provide service to a larger number of users.

- $\quad$ providing adequate quality of service for a greater variety of applications.

- allowing for increased mobility either through roaming or handoff.

- meeting the demand for greater portability by lowering power consumption or increasing battery efficiency.

- offering improved security for devices that use different services.

\section{Technological Integration}

Traditional broadband cable networks require a large capital investment. Wireless technology, on the other hand, is less expensive and much quicker to employ. The primary advantage of wireless networks, however, is its support of mobile applications [9].
Figure 1 provides a general outline of how WiFiWiMAX Technologies are integrated. A WiFi/WiMAX gateway can connect the users of both technologies seamlessly. In this case, the WiFi/WiMAX gateway implements both technologies, permitting users from one side to accesses services on other side. In other words, WiFi users can have access to WiMAX services that are not in their coverage area by employing the multi-hop characteristics of Mobile Ad Hoc Networks (MANET) networks.

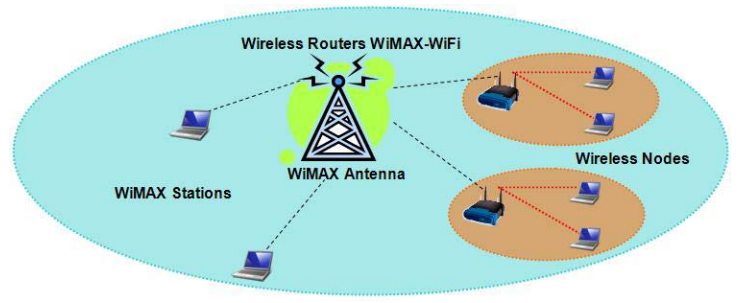

Figure 1: WiFi and Fixed WiMAX
network convergence.

The integration of WiFi-WiMAX has become increasingly common in urban areas where they work in tandem to provide mobile services and a variety of applications. Presently, several cities are attempting to become "wireless cities" in an effort to provide broadband wireless internet access throughout their entire metropolitan areas. WiFi and WiMAX are two options for internet access in metropolitan networks [9]. Presently, integrating WiFi and Fixed WiMAX is the most practical way to deploy large-scale wireless networks in cities that require wireless broadband connectivity [9]. The most famous Secure Wireless Cities (SWCs) projects include Wireless Philadelphia, the San Francisco Techconnect Project, and Google WiFi Mountain View [10, 11, and 12].

The purpose of the Wireless Philadelphia Project is to provide the Philadelphia metropolitan area with wireless services. Handled through Wireless Internet Partners (WIP), even though the entire city does not enjoy full coverage, the goals set by WIP will soon be reached. Although the WIP does not offer its services free of charge, there are some free zones located in public spaces like parks and gardens [10]. San Francisco Techconnect is an initiative for promoting internet services, training, and technical support for the citizens of San Francisco, California. This project places special emphasis on serving low income groups or people with special needs. An important goal of the San Francisco Techconnect project is to promote new applications, contribute to economic growth, and improve municipal services. [11]. The Google WiFi Mountain View project provides free internet to the 
city of Mountain View, California. The main goal is to provide city-wide service and uses mesh architecture to provide WiFi services [12]. Each project was motivated and developed for different reasons, but most of these projects remain true to offering free internet services to entire cities.

The creation of a protocol which allows users access to both types of technologies without problems has great advantages for both users and service providers. Offering integrated WLAN/WMAN services will provide users both performance and high speed data transmission [6].

Ali-Yahiya et al. propose an architecture where the WiFi and WiMAX networks and their traffic routes are separated by dedicated gateways to provide interconnectivity [6]. The main characteristic of this architecture is that it employs an overlapping area between a WLAN and a WMAN hotspot, where they interconnect at a BS and an AP. The WMAN coverage area expands using the WLAN hotspots. More than one AP can be located near the limit of the WMAN's coverage area. The authors assume that WiFi network APs are connected to the WiMAX network by means of a gateway that permits bidirectional interconnectivity. [6].

To better integrate WiFi and WiMAX technologies, it is important to identify the differences between them, including:

- WiFi channels use a specific band frequency while WiMAX permits users to select the channel requirements.

- WiMAX uses licensed frequencies while WiFi employs unlicensed ISM frequency bands.

- WiFi has a range of approximately $10 \mathrm{~m}$ while WiMAX can cover several kilometers.

- WiMAX is a MAN protocol that provides an alternative to DSL and cable modem technologies, providing broadband access for the last mile as it acts as a backbone for WiFi hotspots.

Quality of service (QoS) and mobility are the most common challenges, thus require specific protocols to integrate different types of wireless networks. The most significant problem in terms of QoS is the actual handoff, where nodes must pass information between cells.[6].

An important aspect to consider is that the basic support for QoS differs significantly between WLAN and WMAN because of the different architectures, and more specifically, the specifications of their physical and MAC layers [6].

WiMAX technology supports both PMP and Mesh. A WiMAX PMP network provides last mile access to broadband Internet services by organizing the nodes in a manner that is similar to cell phone networks because it uses a BS. Meanwhile, in mesh topology, an ad hoc network functions independently of the BS. Each node can simultaneously transmit and receive information from neighbor nodes. Additionally, they can send information using a multihop strategy to communicate with nodes that are further away. [13].

The security mechanism of the IEEE 802.16 standard focuses principally of MAC layer security which does not, however, provide sufficient security for multihop transmissions. Consequently, the IEEE 802.16 standard is insufficient to meet the requirement of WiMAX Networks [13].

The goal of our algorithm is to afford users of ad hoc network broadband service using devices that can select the best route in terms of bandwidth, battery residual energy and distance. Figure 2 shows how a WiFi node accesses a WiMAX network.

The proposed protocol can carry out the necessary hops to send and receive data within a WiMAX network. If, a connection is broken, the algorithm is designed to automatically reconnect. Figure 3 provides an example of a service connection from a WiFi node physically located inside a MANET network that requests broadband services from the WiMAX network.

The algorithm is based on the Ad-hoc On Demand Distance Vector (AODV) protocol modeled in OPNET. The following scenario presents a node that functions as a $\mathrm{BS} / \mathrm{AP}$ gateway.

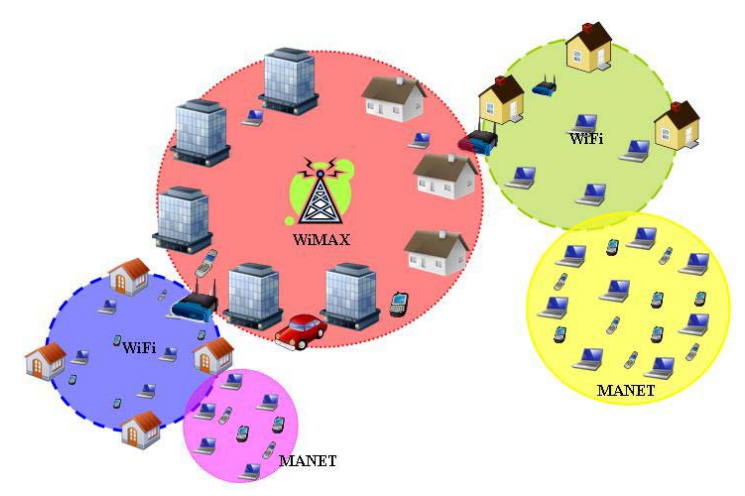

$\begin{aligned} & \text { Figure 2: } \text { Node connectivity from WiFi } \\ & \text { to WIMAX. }\end{aligned}$

AODV is a routing protocol that is used to connect mobile nodes in Ad hoc networks. Important characteristics of AODV include: quick adaptation to dynamic conditions, rapid data processing speed, low network utilization, and its unicast route mecanism that permits easier data transmission between origin and destination pairs [14]. 


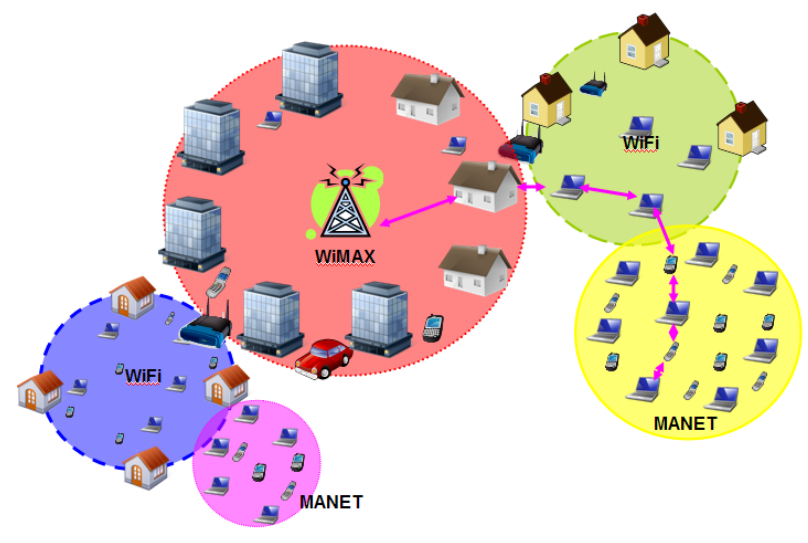

Figure 3: Service Connection from
WiFi to WiMAX.

The main characteristics of AODV algorithm is its reactive route discovery mechanism, that begins only when a node requires information from neighbor nodes. This strategy allows AODV to reduce the number of control packets sent, thus reducing overhead. AODV also maintains dynamic routing tables and its routing mechanism responds quickly to broken links by establishing alternative routes [14].

Figures 4 and 5 show the flow diagrams for the proposed routing algorithm. Source node requesting transmission begins with a RREQ packet until it can reach a destination node or the $\mathrm{WiFi} / \mathrm{WiMAX}$ gateway.

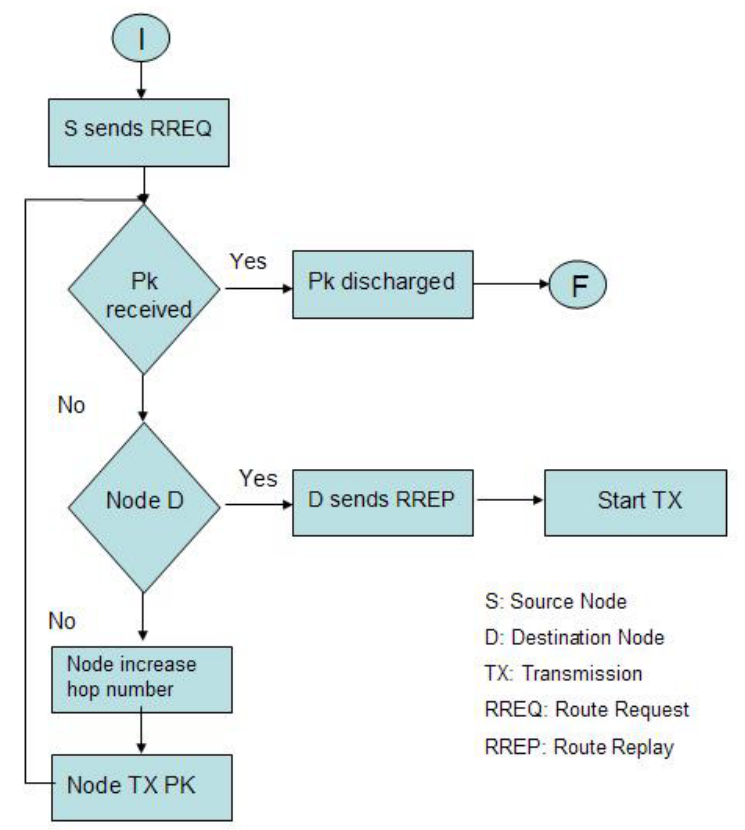

Figure 4: Flow Diagram of the route discover for AODV protocol.
If the destination node is outside of the coverage area of the WiFi network, the AP should retransmit the RREQ packet. After, the WIFI/WIMAX gateway or destination node receive the packet, it will reply a RREP packet to the source node.

\section{Results and Future Work}

Currently, OPNET includes simulation models of WiFI and WiMAX technologies that are compatible with the 802.11 and 802.16 standards in their physical and MAC layers. Furthermore, OPNET also includes the recently modified AODV protocol that was used to develop a new protocol that better integrates WiFI and WiMAX technologies.

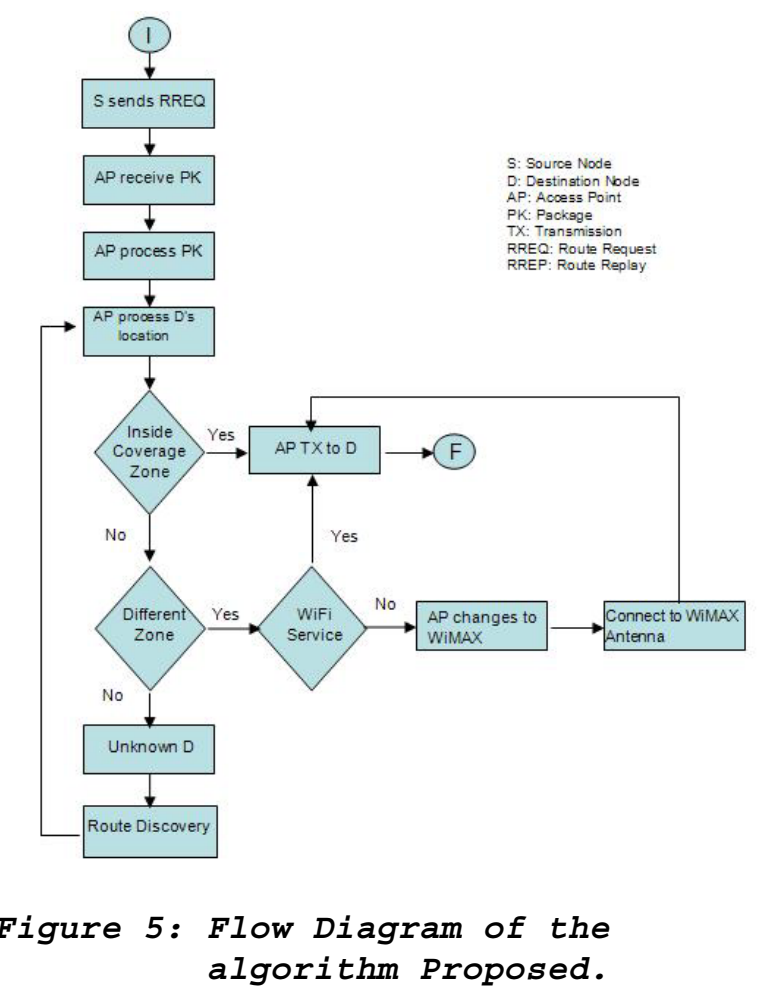

In the future, other algorithms can be developed using the same simulation scenario under different conditions in order to obtain quantitative data to determine which protocol best favors the integration of WiFi and WiMAX technologies.

The integration of AODV with hybrid WiFiWIMAX network will allow the development of a protocol that satisfies performance objectives. The further development of this algorithm will permit the creation and implementation of a protocol based on AODV which will enable any user to seamlessly function within a wireless ad hoc network that employs both WiFi and WiMAX technologies. This integration will, in the future, permit ubiquitous communications 
and data transfer as users move freely from one technology to the other.

\section{References}

[1] Y. Tara Ali,, B. André-Luc, P. Guy. "Policy-Based Threshold for Bandwidth Reservation in WiMax and Wifi Wireless Networks," En Proc. 2007 Wireless and Mobile Communications. ICWMC '07. Third International Conference on, pp.76.

[2]R. Bera, B. Paul, A. Guchhait, S. Sil. N.B. Sinha, y S. Dogra. "Wireless Embedded System for Multimedia Campus Network Utilizing IEEE $802.11 \mathrm{~N}$ (draft) and WiMax Radio," en Proc. 2007 Wireless and Optical Communications Networks, pp.1-5.

[3]S. Jindal, A. Jindal, y N. Gupta. "Grouping WI-MAX, 3G and WI-FI for wireless broadband," en Proc. 2005 First IEEE and IFIP International Conference in Central Asia.

[4] A. Ian F., W. Xudong, W. Weilin. "Wireless Mesh Networks: A Survey, "Computer Networks, Volume 47, Issue 4, 15 March 2005, pp. 445-487.

[5] T. Su-En. "WiMax-Prospect and New Business Models, " en Proc. $20053 G$ and Beyond, $20056^{\text {th }}$ IEE International Conference on, pp.1-5.

[6]T. Ali-Yahija, K. Sethom, y G. Puiolle. "Seamless Continuity of Service across WLAN and WMAN Networks: Challenges and Performance Evaluation," Proc. 2007 IEEE/IFIP International Workshop, pp. 1-12

[7] S. Dhawan. "Analogy of Promising Wireless Technologies on Different Frequencies: Bluetooth, WiFi, and WiMAX," en Proc. 2007 Wireless Broadband and Ultra Wideband Communications, 2007. AusWireless 2007. The 2nd International Conf.

[8] J.G. Andrews, A. Ghosh y R. Muhamed. "Introduction to Broadband Wireless," en Fundamentals of WiMAX, Understanding Broadband Wireless Networking. Ed.Prentic Hall, 2007, pp 3-32.

[9] Y. Chen. "Achieve User Authentication and Seamless Connectivity on WiFi and WiMAX Interworked Wireless City," en Proc. 2007 Wireless and Optical Communications Networks, pp. 1-5
[10]
Wireless http://www.wirelessphiladelphia.org/

[11] San $\quad$ Francisco
http://www.sfgov.org/techconnect/

[13]K. Lu, Y. Qian, H. Chen. (2007, Mayo). A Secure and Service-Oriented Network Control Framework for WiMAX Networks. IEEE Communications Magazine, pp. 124- 130

[12] Google WiFi, http://wifi.google.com/ 